\title{
La investigación sobre los horrea de época romana: Balance historiográfico y perspectivas de futuro.
}

\author{
Javier Salido Domínguez \\ Universidad Autónoma de Madrid
}

\section{Resumen}

Este trabajo se centra en el análisis de la evolución historiográfica de la investigación sobre los horrea romanos con el fin de comprender el contexto histórico en que se plantearon las principales teorías de los siglos XIX y XX. Además se pretende presentar propuestas de estudio para profundizar en el estudio de este tema que resulta imprescindible para comprender la economía en época romana.

Palabras Clave: historiografía, graneros, horrea, campamentos, villae, época romana.

\begin{abstract}
This investigation presents the analysis of the historiographical evolution of the research about the Roman horrea in order to understand the historical context in which the first theories of the XIX and XX century were formed. Moreover, the investigation presents certain study proposals that would amplify the analysis of this subject, which is indispensable to the understanding of the economy of the Roman Empire.
\end{abstract}

Key words: historiography, granaries, horrea, fortress, villae, Roman times.

EL INTERÉS POR EL ESTUDIO DEL APROVISIONAMIENTO DE GRANO A ROMA: FINALES DEL SIGLO XIX-COMIENZOS DEL SIGLO XX

Los primeros trabajos dedicados al estudio del grano en época romana se centraban en la investigación de los mecanismos de administración, organización y control del cereal por parte de Roma y el aprovisionamiento de grano a la capital. La causa que explica el interés por centrar el estudio en la capital del Imperio romano no es

1 Este trabajo se ha desarrollado en el marco del proyecto de investigación Formación y disolución de civitates en el NO peninsular. Estructuras de poblamiento y territorio otro que la limitación de las fuentes documentales con las que contaban los grandes historiadores de finales del siglo XIX y comienzos del siglo XX. En este periodo, la principal fuente utilizada para abordar el estudio de la antigüedad son los textos clásicos y para analizar la administración y suministro de grano a Roma, la obra por excelencia es el Codex Theodosianus. Se trata de una labor encomiable e importante porque no sólo supone el inicio y acercamiento a uno de los principales temas económicos de la Antigüedad, sino que

(HR2008-06018-CO3/HIST), financiado por el Ministerio de Ciencia y Tecnología. La investigadora principal es la Dra. Carmen Fernández Ochoa. 
también presentaron una detallada interpretación filológica de los pasajes referidos al asunto. La crítica que se puede plantear a estos análisis es la escasa atención que se presta a otro tipo de fuentes ya conocidas, como los testimonios arqueológicos, las inscripciones, representaciones en el arte, etc. Después de varios trabajos será R. Cagnat quien inicie este trabajo que podemos calificar como "multidisciplinar".

Entre las grandes obras de inicios del siglo XX a las que me he referido, se pueden destacar entre otras el artículo publicado en 1870 por Hirschfeld titulado Die Getreideverwaltung in der römischen Kaiserzeit, que tiene el valor de analizar la burocracia que forma parte de la administración del aprovisionamiento de grano no sólo en época bajoimperial, sino también en periodos anteriores. Poco tiempo después, G. Krakauer publicó su tesis en 1874 bajo el título Das Verpflegungswesen der Stadt Rom in der späteren Kaiserzeit, en el que aborda el estudio del aprovisionamiento no sólo de grano sino también de otros productos, como el aceite, vino y carne. Aunque el libro contiene información muy valiosa, es cierto que se limita a presentar una recopilación de fragmentos de textos clásicos ya conocidos. Ésta será precisamente la crítica que formulará a dicha obra otro de los grandes historiadores de este periodo, E. Gebhardt quien dedicó su tesis doctoral de 1881 a la misma cuestión; su obra fue publicada bajo el título Studien über das Verpflegungswesen von Rom und Constantinopel in der späteren Kaiserzeit. Al mismo tiempo que se analiza el asunto desde el punto de vista administrativo, comienza a surgir la necesidad de estudiar la cuestión legal, como Babled quien trata el asunto en su obra De la cura annonae chez les Romains, impresa en 1892.

El cambio de siglo supone la incorporación de nuevas fuentes documentales que permiten analizar el asunto con mayor profundidad. El primero en iniciar esta tendencia es el francés Cagnat quien en el artículo L'annone d'Afrique se centra en la investigación del aprovisionamiento de grano a Roma desde las provincias del norte de África.

El valor de estas obras reside en la necesidad de analizar la economía romana a partir de las fuentes de las que disponían y supone el inicio de la investigación de un tema inexistente en la historiografía anterior que subraya la importancia que tuvo el grano en el Imperio y la eficacia de la organización de un producto de primera necesidad por parte de la administración. Esta trascendencia tiene su repercusión en la publicación de obras que pretenden dar continuidad a estos trabajos y plantear nuevas cuestiones. Entre estas publicaciones, se puede destacar el libro de Chastagnol, La préfecture urbaine à Rome sous le Bas-Empire (1960), la tesis de Alzon, Problèmes relatifs à la location des entrepôts en Droit romain (1964), el trabajo de E. Tengström, Bread for the People (1975) y más recientes como la publicación de Durliat, De la ville antique à la ville byzantine le problème des subsistances (1990), la tesis de Jaidi, L'Afrique et le blé de Rome aux IVème et Vème siecles (1990) o las actas de los coloquios Le ravitaillement en blé de Rome et des centres urbains des débuts de la République jusqu'au Haut Empire (1994) y Nourrir les cités de Méditerranée antiquité-temps modernes (2003).

LA EXCAVACIÓN DE OSTIA Y LA RENOVACIÓN EN EL ESTUdIO DE LA ECONOMÍA ROMANA: MEIGGS, RICKMAN Y LOS GRANDES COMPENDIOS DE LA DÉCADA DE LOS "70

Los descubrimientos arqueológicos supondrán una verdadera renovación en el estudio de la Antigüedad y el conocimiento de los graneros de Ostia pondrá de manifiesto que es imprescindible investigar el aprovisionamiento de grano a partir de la información arqueológica. Anteriormente se centraba el interés en analizar cada una de las fases que requería la producción, almacenamiento, transporte y distribución del grano; en este momento, se empiezan a conocer los edificios en los que se desarrollaban estas actividades. Si antes no era posible detectar arqueológicamente el transporte y consumo de grano, ahora los almacenes constituyen el mejor testimonio de la existencia de una red de exportación del cereal desde las provincias. Si hay que citar a un especialista que inicie el estudio general de los graneros en el Imperio, habría que referirse sin duda a Russell Meiggs quien en 1960, a la edad de 58 años, publicó su primera gran obra, Roman Ostia, en el que recopila la información de 35 años de trabajo que inició siendo un recién licenciado en 1925. La originalidad de Meiggs se debe al contexto histórico en el que vivió. Forma parte de una generación de grandes especialistas de la Antigüedad formados en la universidad de Oxford que, junto a Dunbabin, Chilver o Jones, muestran una preo- 
cupación por las realidades de la sociedad griega y romana más allá del mundo de la política y la literatura. Este enfoque empírico y positivista provoca que el autor base sus conclusiones en el conocimiento personal y en su experiencia. Su positivismo y su interés en el funcionamiento de la sociedad no son productos de la tradición o de la investigación en la biblioteca, sino que proceden de la propia realidad que están viviendo (Murray, 1996: 9). Muchos de estos historiadores y compañeros de su generación tuvieron que abandonar la investigación y dedicarse a la organización de un país preparado para la guerra. En el caso de Meiggs, se vio obligado, durante la Segunda Guerra Mundial, a trabajar en el Ministerio de Abastecimiento ("Ministry of Supply"), donde desde 1941 desempeñó el puesto de jefe del departamento de provisión de madera ("Chief Labour Officer of the Home Timber Production Department of the Ministry"). Sin duda, este trabajo que suponía controlar la producción y transporte de la madera influirá en su obra arqueológica, especialmente en un trabajo que trata sobre la administración en época romana del aprovisionamiento de madera (Trees and Timber in the Ancient Mediterranean World, 1982).

La obra de Meiggs adquiere más importancia si tenemos en cuenta la repercusión de los datos arqueológicos aportados durante los años de excavación en el puerto de Roma, la validez de la documentación que nos legó, pero sobre todo, la herencia intelectual que permitió abrir una línea de investigación pujante que tiene su reflejo en la publicación de cuantiosas obras dedicadas al estudio de los horrea romanos. No es extraño que sea precisamente Meiggs quien formara parte del tribunal de tesis de G. Rickman quien acabará publicando la mayor obra de referencia realizada hasta el momento sobre los sistemas de almacenamiento en el occidente del Imperio romano. El trabajo inicial realizado entre 1958 y 1962 se presentó bajo el título de The design, structure and organisation of horrea under the Roman Empire, pero no será publicado hasta 1971 cuando sale a la luz el libro Roman granaries and store buildings. En ésta, se incluye el estudio de los horrea excavados en Ostia y en otras provincias del occidente romano, diferenciando entre los graneros de ámbito civil y militar. La información con la que contaba era original en muchos casos, pues el principal arqueólogo dedicado a la excavación de numero- sos campamentos romanos de Inglaterra, Sir Ian Richmond, era precisamente su director de tesis. La contribución de Rickman es admirable, pues realiza un examen perfecto de la información aportada por los autores clásicos y recopila toda la información existente hasta el momento sobre las técnicas constructivas de este tipo de edificios con el fin de definir perfectamente los elementos propios de los horrea, los tipos diferenciados según su planta y las variedades regionales. La crítica que se puede hacer a dicha monografía es la ausencia de un corpus de graneros conocidos en el que se detalle la cronología, región geográfica, dimensiones y técnicas constructivas de cada uno, aunque no se puede desestimar la habilidad de exponer los datos generales con el fin de determinar cuáles son las técnicas propias de este tipo de edificaciones.

Es importante tener en cuenta que en el momento de iniciar su tesis doctoral, Rickman ya contaba con documentación de memorias antiguas de excavaciones y con nuevos datos sobre las intervenciones que se estaban desarrollando en ese momento en diversos campamentos romanos de Britannia, Germania e Hispania. Sin embargo, será el descubrimiento de los horrea de Ostia el motor que impulsó el estudio de los almacenes del occidente del Imperio y los medios administrativos para controlar la producción, almacenaje y transporte del grano en el Imperio.

En Gran Bretaña son numerosos los ejemplos de graneros militares excavados a principios del siglo XX. Los primeros asentamientos militares en los que se descubrieron horrea formaban parte del Muro de Antonino. Con ello, me refiero a Lyne (Christison y Anderson, 1901), Castlecary (Buchanan, 1903), Rough Castle (Buchanan, 1905), Bar Hill (Macdonald y Park, 1906), Cappuk (Stevenson y Miller, 1912), Balmuildy (Miller, 1922), Old Kilpatrick (Miller, 1928), Mumrills (Macdonald y Curle, 1928-1929) y Cadder (Clarke, 1933). En una fecha más reciente, se inician diversas campañas de excavación en varios campamentos romanos pertenecientes al Muro de Adriano, como Corbridge (imagen 1) (Knowles y Forster, 1910), Housesteads (Bosanquet, 1904), Rudchester (Brewis, 1925), South Shields (Richmond, 1934; 1953), Brampton Old Church (Simpson y Richmond, 1936), Chester (Petch y Thompson, 1959), Haltonchesters (Gillam, 1961). A mediados del siglo XIX, John Clayton descubrió y posterior- 
mente demolió dos graneros en la retentura del campamento de Chesters. La información de las que disponemos fue publicada posteriormente por Birley (Birley, 1960; 1961). Estas intervenciones arqueológicas permitieron constatar que los graneros del Muro de Adriano fueron construidos con técnicas similares a los horrea del Muro de Antonino. Al mismo tiempo, la excavación de yacimientos en Gales y Escocia permitió conocer más edificios de este tipo y apreciar unos criterios comunes en su construcción, aunque correspondiesen a regiones y épocas diferentes. En Gales, se pusieron al descubierto almacenes en el campamento de Pennydarren (James, 1906), Gellygaer (Ward, 1903; Haverfield, 1910), Castell Colen donde se iniciaron excavaciones a principios de siglo XX y se retomaron posteriormente (Evelyn-White, 1914; Alcock, 1954-1957), Caernarvon (Wheeler, 1923) y Caerhun (Reynolds, 1938). También en Escocia, como Fendoch (Richmond y Mcintyre, 1939). Poco antes de la publicación de la obra de Rickman, en 1968 se dieron a conocer los resultados de las excavaciones del campamento de Richborough iniciadas en la década de los años '20 y '30 del siglo XX (Cunliffe, 1968), Hod Hill (Richmond, 1968), en los campamentos auxiliares de Pen Llystyn (Hogg, 1968) y poco después las excavaciones desarrolladas entre 1961 y 1969 en Fishbourne (Imagen 2) (Cunliffe, 1971). Respecto a Inchtuthil, en este momento Rickman solamente contaba con datos limitados de los graneros que se estaban excavando en el campamento legionario. Las campañas de excavación en este campamento que se prolongaron desde 1952 hasta 1965 fueron en parte publicadas en 1959 por el director de tesis del doctorando Rickman, I.A. Richmond (Richmond, 1959) y no verán la luz hasta 1985 cuando se publica una monografía extensa sobre los restos encontrados (Pitts, Joseph, y Cowie, 1985).

En Germania, también eran numerosos los graneros conocidos, pero Rickman no hace referencia a tantos ejemplos como para el caso de Britannia. Los graneros que menciona son un total de diecinueve y en la actualidad se conoce por lo menos el doble. Las memorias de excavación publicadas, especialmente las correspondientes a la serie Der obergermanisch-raetische Limes des Römerreiches, ofrecían datos muy concretos de los almacenes y graneros de los campamentos. Entre éstos, se hallan los horrea excavados en Suiza como Vindonissa (Laur-Belart,
1935), los Países Bajos como Nijmegen (Brunsting, 1961; Bogaers, 1967) y mayoritariamente en Alemania, como Ruffenhofen (Kohl, 1896), Wiesbaden (Reuter, 1871; Ritterling, 1909), Pfünz (Winkelmann, 1901), Okarben (Wolff, 1902), Benningen (Mettler, 1902), Neuss (Koenen, 1904), Urspring (Fabricius, 1904), Theilenhofen (Eidam, 1905), Kapersburg (Jacobi, 1906), Weissenburg (Fabricius, Kohl y Tröltsch, 1906), Bonn, Hofheim (Ritterling, 1913), Saalburg (Jacobi, 1897; 1937), Hüfingen (Revello, 1937), Niederbieber (Ritterling, 1937), Haltern (Stieren, 1943), y Rödgen (Schönberger, 1963-1964). Este amplio elenco de información contrasta con la documentación que se publica para el caso de Hispania, caso que analizaremos separadamente. La información se limita a la publicación de los resultados de las excavaciones arqueológicas dirigidas por Schulten en los campamentos romanos de la región septentrional de España, donde se pusieron al descubierto los graneros de época republicana mejor conservados del Imperio.

Al analizar el momento de publicación de la mayoría de los graneros militares excavados en Britannia, Germania e Hispania, podemos llegar a la conclusión de que, aunque la documentación relativa a los almacenes militares era ingente ya en la primera mitad del siglo XX, no fue hasta las excavaciones de Meiggs en Ostia cuando se decide investigar el tema de un modo general. Esto además permite analizar tanto los horrea de ámbito civil como militar en una misma monografía. El propio Rickman reconoce en el prólogo de su obra que son las excavaciones de Ostia las que aportan el mayor conocimiento sobre los almacenes romanos y son las memorias de Meiggs una referencia fundamental en su libro: "Since then our knowledge has been greatly increased, particularly by the excavations at Ostia just before the Second World War" (Rickman, 1971: XVII). Es probable que el conocimiento de los almacenes de Roma y su puerto motiven el estudio de los graneros del ámbito occidental del imperio debido al peso de la historiografía anterior volcada al estudio del aprovisionamiento de grano desde el resto de provincias a la capital. De hecho, el interés de Rickman se centrará después precisamente en el estudio de los mecanismos que emplea la administración romana desde la etapa republicana hasta la época bajoimperial para la provisión de grano a Roma, contando con los datos arqueológicos ya expuestos en su obra de 1971. El resulta- 
do será la publicación de su segunda gran obra titulada The corn supply of Ancient Rome (1980).

La aparición de la obra de Rickman animó a otros investigadores a emprender el estudio de los graneros. Será en esta década cuando se publican todas las monografías dedicadas al análisis de los edificios de almacenaje de cereal, aunque con un inconveniente, todos se centran en el mismo ámbito geográfico, Britannia. Cuatro años después de la obra de Rickman, Manning decide mejorar un capítulo que se hallaba incompleto en la obra del anterior, el estudio de los graneros militares construidos en madera. En el artículo Roman military timber granaries in Britain, no sólo presenta un compendio de todos los datos de este tipo de almacenes, sino que supone un avance notable en la investigación al proponer por primera vez los datos de cuantificación relativos a la capacidad de cada uno de los edificios excavados. También se puede considerar una ampliación de la obra de Rickman y un progreso en el conocimiento de estas estructuras la recopilación de datos relativos a los graneros militares construidos en piedra publicada por Gentry. Siguiendo la metodología empleada por Manning, escribe Roman military stone-built granaries in Britain (1976), donde establece también diferentes tipos de edificios y plantea el cálculo de capacidad con una ventaja con respecto a la anterior, pues cuenta con las dimensiones internas de los edificios y, por tanto, sus datos se pueden aproximar más a la realidad. En este sentido, es interesante destacar la ausencia de este tipo de estudios en otros ámbitos del Imperio, como Germania o Gallia. Los grandes graneros de madera y piedra de los campamentos del limes renano-danubiano se conocían en estos años $\mathrm{y}$, sin embargo, ningún investigador decidió iniciar el estudio de estos edificios. Unos años más tarde, en 1980, Petculescu presentó un estudio de conjunto de los horrea de la Dacia (Roman Military Granaries in Dacia). El interés de este trabajo se halla en los datos que se indican, pero apenas aportan novedades en cuanto a la metodología empleada.

Así pues, podemos concluir que en la década de los '70 se publican compendios que completan la obra de Rickman y en éstos se presentan avances en cuanto al tipo de datos como la capacidad, relación con otras construcciones del campamento, etc., pero no se da importancia al contexto histórico en el que se construyeron. No es extraño que sean los graneros militares las construcciones analizadas en primer lugar, pues son los edificios de los que las excavaciones arqueológicas estaban aportando más información. El estudio de los graneros militares, que indican todas las técnicas de construcción y elementos propios de este tipo de estructuras, permite a partir de este momento concretar los criterios a seguir para diferenciar estos almacenes de otros edificios de funcionalidad agrícola.

\section{DEL ESTUDIO DE LOS GRANEROS MILITARES A LA INVESTIGACIÓN DE LOS HORREA RURA- LES}

Como indiqué anteriormente, el estudio de los campamentos romanos permitió conocer las técnicas empleadas en la construcción de los almacenes destinados al almacenaje de cereal. El reconocimiento de este tipo de construcciones en el interior de los campamentos resultó siempre más sencillo que en yacimientos de ámbito civil. En primer lugar, las fuentes escritas aportan mucha información sobre el aprovisionamiento de grano al ejército y los tipos de productos que se almacenaban en el interior de estas construcciones (cereal además de víveres como carne, legumbres, etc.). El hecho de que la mayoría de los campamentos contase con este tipo de edificios nos permite establecer además tipologías, conocer la estructuración interna de los edificios, su ubicación y datos relativos a espacios porticados, entradas y otros elementos como zanjas de drenaje, contrafuertes, etc., información que posibilita el reconocimiento de estos edificios en el ámbito civil. Esto explica que los grandes compendios de graneros militares se conviertan en referencia de las monografías que se publican a finales de los años ' 70 centradas en el reconocimiento y estudio de los graneros rurales romanos. Es curioso que, en este caso, el estudio de los horrea rurales sobrepasa las fronteras de Gran Bretaña e impulsa la investigación en otros países europeos.

En 1978, Rossiter inicia el estudio de los almacenes en las villae de Italia. En su obra Roman farm buildings in Italy, analiza el granero de la villa de Russi e incluye este tipo de construcciones en un capítulo dedicado a horrea, espacios de almacenaje y corrales. Más ambicioso es el proyecto de Morris quien publica en 1979 Agricultural Buildings in Roman Britain, monografía que trata de diferenciar varios tipos de 
horrea de ámbito rural. Se aborda esta investigación distinguiendo entre graneros y otros edificios de funcionalidad desconocida, entre los que destacan varias construcciones que albergan en su interior hornos que define como secaderos de grano (corn drying ovens). Esta especialista llega a la conclusión de que el mejor testimonio para interpretar un edificio, además de su ubicación, calidad de materiales y otras técnicas de construcción, es la existencia de un pavimento sobreelevado (tabulatum). Morris diferencia los graneros de estilo militar (categoría 1) que cuentan con un sobrado de madera apoyado sobre postes (1.1), apoyos de madera hundidos en el interior de varias zanjas (1.2), muros de madera (1.3), paredes de piedra (1.4) o pilae (1.5) y un segundo tipo (categoría 2) que también disponían de un suelo de madera, pero sujeto a las paredes de las estancias. En éstos últimos, se supone la existencia de vigas de madera que apoyan sobre pequeños pilares anexos a las paredes o se insertan en el interior de los mechinales o huecos realizados en la pared para sostener este entramado de madera. Sin duda, la obra de Morris es la mejor monografía dedicada al estudio de los edificios de funcionalidad rústica que se ha publicado hasta el momento, pues trata de presentar hipótesis sobre el destino de algunas estructuras, establece una tipología muy útil para diferenciar los graneros excavados y plantea muchas dudas sobre el uso de determinadas construcciones que aún no se han podido resolver.

Ante la necesidad de dar respuesta a muchas cuestiones propuestas en estos trabajos, algunos autores se plantean qué tipos de cereales eran almacenados en época romana, qué sistemas de almacenaje se empleaban y cómo se guardaba. Para ofrecer una explicación de estos planteamientos, fue necesario en muchas ocasiones recurrir al estudio de los tipos de almacenes utilizados por comunidades actuales y analizar los restos materiales que podrían permanecer en caso de ser derrumbados o abandonados. En este contexto, se debe reconocer el esfuerzo del francés François Sigaut quien pretendió promover este tipo de estudios. La diferencia estriba en que no se trata de presentar un corpus documental de la información aportada por las excavaciones, sino que pretende ofrecer información procedente de la etnoarqueología y la arqueología experimental con el fin de resolver cuestiones aún no planteadas. Para ello, promueve junto a Gast la organización de dos congresos en 1979 y 1981 conocidos como Les techniques de conservation des grains à long terme. Leurs rôles dans la dynamique des systèmes de culture et des sociétés y un tercero en 1985 en el que también colabora Beutler. En el segundo encuentro, Sigaut presenta el trabajo Identification des techniques de conservation et de stockage des grains, en el que ya plantea hipótesis de trabajo que irá analizando en aportaciones posteriores, como el artículo A method for identifying grain storage techniques and its application for European Agricultural History (1988-1991). En estos estudios, Sigaut diferencia tres tipos de almacenes: las técnicas de conservación en atmósfera confinada (los silos) y sin control atmosférico específico (recipientes cerámicos), donde se guarda el cereal a granel y las técnicas de atmósfera controlada y renovada (graneros sobreelevados), donde se almacena el grano en espigas. También plantea un dato interesante que también hay que tener en cuenta, la capa de cereal almacenado tenía que tener un espesor máximo de $1 \mathrm{~m}$. para evitar su fermentación. Estas conclusiones permiten cuestionar los cálculos de capacidad planteados por Gentry y Manning para los horrea militares.

La publicación de estos trabajos supone la aparición de obras que, continuando con la tradición de plantear tipologías, tienen además en cuenta cómo se debía almacenar el grano y otros sistemas de almacenaje como los silos. En esta línea, hay que destacar dos estudios dedicados al análisis de los graneros excavados al norte de la Gallia. La primera obra de referencia es publicada por Demarez en 1987, bajo el título Les bâtiments à fonction économique dans le fundi de la Provincia Belgica. Demarez incluye los silos en una primera categoría (tipo 0) y diferencia cuatro tipos diferentes: las construcciones idénticas a los graneros militares (I), los edificios rectangulares, generalmente con contrafuertes, que cuentan con uno o varios espacios interiores (IIa), edificaciones rectangulares con una mayor compartimentación interna (IIb), graneros en forma de torre o Speicherturm (III) y los edificios de planta basilical (V). La principal diferencia con las tipologías que se habían planteado es la inclusión de la posibilidad de que se siguiesen excavando silos de almacenaje de grano en época romana y la existencia de dos tipos nuevos de graneros propios de 
esta región geográfica: las torres-silos ${ }^{2}$ y otros edificios de planta basilical ${ }^{3}$. Cinco años más tarde, en 1992 Van Ossel publica su tesis doctoral sobre los Établissements ruraux de l'Antiquité tardive dans le Nord de la Gaule, en el que analiza la información aportada por las excavaciones de un modo crítico y trata de aunar todas las tipologías propuestas en la siguiente clasificación: edificios con suelo sobreelevado (tipo I), las torres-silos (II) y edificios que reaprovechan los restos materiales de construcciones anteriores (III).

La importancia que estos trabajos comienzan a ofrecer sobre la necesidad de analizar los graneros romanos como edificios que nos aportan información valiosa de la economía de los establecimientos rurales se ve reflejada en la publicación de análisis meticulosos de este tipo de estructuras en regiones concretas. Tal es el caso de la monografía publicada por Busana en el año 2002 sobre las Architetture rurali nella Venetia romana, el trabajo del mismo año realizado por Ursula Heimberg, Römische Villen an Rhein und Maas y la última publicación sobre los almacenes del área de Mauritania Tingitana, a cargo de Papi y Martorella (2007).

\section{DEL CONTINENTE AL CONTENIDO: ESTUDIOS ARQUEBOTÁNICOS Y PALEOAMBIENTALES}

Hasta finales de los años '70 solamente los restos constructivos permitían interpretar los edificios como graneros, pero el desarrollo de nuevas técnicas de observación, la consolidación de los estudios interdisciplinares y la investigación genética hicieron posible transformar la simple identificación taxonómica en intentos de interpretación de las interrelaciones entre las comunidades humanas y las plantas (Buxó, 1991: 25). Esta disciplina estuvo generalmente ligada al estudio de la reconstrucción paleoambiental de la Prehistoria, pero paulatinamente se fue aplicando a otros periodos, como la época romana.

2 Años antes se habían puesto al descubierto edificios conocidos como Strebepfeilerbau que son construcciones de planta rectangular dotadas de contrafuertes, similares a los graneros militares. Las publicaciones de las excavaciones arqueológicas desarrolladas en el yacimiento de Echternach (Metzler, Zimmer y Bakker, 1981) y Wasserbillig (Krier y Wagner, 1983) ofrecen datos sobre las técnicas empleadas en la cons-
Los primeros estudios realizados informan sobre los tipos de cereales almacenados, el estado en el que se encuentran (carbonización, germinación), cómo se guardaría el grano y aportan información sobre los insectos asociados al cereal acumulado. Estos primeros análisis se centraron en el reconocimiento del cereal encontrado en determinados yacimientos y los primeros resultados ya ofrecían datos importantes. Se pudo constatar la existencia de un comercio a larga distancia de cereal desde otras regiones a asentamientos militares y civiles de Britannia, tal y como evidencia la aparición de grano importado. Entre estos primeros trabajos, podemos destacar los análisis de restos botánicos aparecidos en un almacén de época flavia del anexo situado junto al campamento de Caerleon (Helbaek, 1964), en un almacén militar de York datado en los años 70-120 d.C. (Buckland, 1976; Kenward y Williams, 1979) y los estudios de los restos aparecidos en un granero del siglo III d.C. de South Shields, que fue utilizado como centro de aprovisionamiento de cereal para el ejército destinado a reconquistar el territorio escocés (Van der Veen, 1988). En otras regiones, la importación de productos como el arroz también se pudo documentar a partir de la recogida y análisis de los restos botánicos, como en el campamento militar de Neuss (Knörzer, 1970).

El desarrollo de estos análisis puso de manifiesto que la aparición de cereal nos permite interpretar como graneros ciertos edificios que, debido a su estado de conservación, apenas ofrecen datos sobre las técnicas empleadas en su construcción y, por tanto, hubiese resultado imposible confirmar su uso como horrea de no haberse realizado estudios arqueobotánicos. En este sentido, debemos destacar el análisis de Matterne y otros investigadores a partir de las muestras recogidas en el almacén urbano de Amiens (Matterne et al., 1998). La importancia de este trabajo estriba en que no sólo determinó el uso como granero de un edificio que constructivamente no se asemejaba a los horrea romanos, sino que proporciona datos

trucción de este tipo de edificaciones que interpretan como Kornspeicher .

3 La teoría es planteada ya por Petrikovits (Germania 34, 1956: 99-125) que interpreta el edificio IV de la villa de Wollersheim como un granero. La presencia de estos edificios es muy extendida en Gran Bretaña donde se conocen como Aisled House. 
muy interesantes sobre cómo se almacenaba el cereal en su interior, ofrece todo tipo de detalles sobre la organización interna del almacenaje, qué productos se habían almacenado antes que otros y presenta unos datos relativos a la capacidad del granero más aproximados que los planteados anteriormente por Gentry o Manning. Sin duda, la repercusión de este trabajo ha sido notable y actualmente los grandes especialistas sobre el tema usan como referencia los datos aportados para el almacén de Amiens para determinar la capacidad máxima de graneros excavados en otros yacimientos (Van Ossel y Defgnée, 2001: 232-233).

La confrontación de los resultados carpológicos y arqueozoológicos permitió además conocer el proceso de extensión del cultivo de determinados cereales y, analizando regiones amplias, se pudieron establecer las diferencias en el sistema de explotación del territorio entre la edad del Hierro y la época romana, así como determinar las nuevas especies cultivadas en el periodo clásico (Matterne, 2001; Lepetz y Matterne, 2003). La relación entre los restos aparecidos y la explotación del entorno permitió también reconstruir la explotación humana del territorio, reconocer la vegetación y flora de las inmediaciones de los yacimientos e incluso determinar la capacidad productiva cerealística de una región a partir de los datos carpológicos procedentes de varios yacimientos. En esta línea de investigación, se encuentran los estudios realizados por Kooistra para la región de Germania Inferior (Kooistra, 1996).

\section{LA investigación en LA Península IBÉRICA}

La importancia que tiene el estudio de los graneros romanos documentados en la Península Ibérica contrasta con la escasa atención que los especialistas han prestado a este tipo de investigaciones. En la actualidad, los restos constructivos que se hallan en España son un testimonio clave para la comprensión de las técnicas empleadas en la edificación de los primeros graneros construidos en el Imperio romano. Solamente en Hispania se han conservado restos materiales correspondientes a los graneros militares de época republicana y en esta región geográfica es donde se conocen mejor los edificios destinados al almacenaje de cereal de época prerromana. El hecho de que en la Península se construyeran los graneros en piedra ha permitido que nuestro país y la investigación española resulten una pieza clave en el estudio del origen de estas construcciones y, en mi opinión, son la mejor muestra de lo que nuestra arqueología puede aportar a este campo.

Los primeros datos sobre la existencia de graneros romanos construidos en Hispania proceden de las excavaciones arqueológicas desarrolladas por Adolf Schulten entre 1905 y 1912 en el campamento principal de asedio a Numancia, Castillejo III (Soria, Castilla y León) (Schulten, 1927), que según Schulten correspondería al cuartel general de Escipión y a la campaña de los años 134-133 a.C. y en el de Renieblas V (Soria, Castilla y León) (Schulten, 1929; Luik, 2006), que se consideró un campamento de invierno de 75-74 a.C. (Schulten, 1927; Gómez-Pantoja y Morales Hernández, 2002; Morales Hernández, 2006). A pesar de las dificultades económicas del periodo de guerra, la inflación monetaria y la gran crisis en la que España estaba inmersa, se pudo publicar la gran obra de Schulten Ausgrabungen in Numantia entre 1913 y 1931. Desde el punto de vista arqueológico, los resultados de las excavaciones del arqueólogo alemán plantean numerosos problemas de orden metodológico, pues no tenía en cuenta las estratigrafías y para su datación se basa principalmente en la información proporcionada por los textos clásicos sobre el periodo de conquista de la antigua Hispania (Morillo, 1991: 177-178). No se puede dudar de la datación republicana de estos campamentos, pero es necesario tratar de fijar una datación a partir de los datos arqueológicos que supere el "criterio filológico".

Las expectativas generadas por Schulten acerca del estudio de los campamentos numantinos quedaron truncadas con el inicio de la I Guerra Mundial. En los últimos veinte años, se ha producido un notable avance en el estudio de la arqueología militar romana y en el conocimiento de la conquista romana gracias al trabajo de especialistas como Morillo o Quesada. El resultado de este vertiginoso progreso en el estudio del ejército romano en Hispania se ha traducido en la multiplicación de publicaciones que han abierto un interesante debate científico sobre el proceso de conquista romana, aculturación e impacto económico, socio-político y cultural (Morillo, 2002; 2003; 2006a; 2007). Se ha podido precisar la datación de campamentos, como el de Renieblas 
que podría ser coetáneo al asedio y caída de Numantia (Hildebrant, 1979: 266-271; Sanmartí, 1992: 427-428.) y se han publicado interesantes trabajos sobre las redes comerciales y el abastecimiento de productos al ejército en Hispania (Fernández Ochoa y Morillo, 2002; Morillo, 2006b). Sin embargo, no se han reestudiado los graneros militares, testimonio clave para el reconocimiento de este tipo de estructuras de época republicana en el Imperio. Recientemente, Dobson en su revisión de las excavaciones de Schulten en los campamentos que formaban parte de la cicumvallatio de Numantia, señaló la posibilidad de que cuatro edificios próximos a la porta praetoria del fuerte militar de Valdevorrón cumpliesen la función de horrea (Dobson, 2008). Estas estructuras fueron interpretadas por Schulten como los dispositivos donde se situaban las catapultas (Schulten, 1927: 219), aunque ya advirtió de las similitudes que existían con los horrea de los campamentos militares de Castillejo III (imagen 3) y Renieblas V (Schulten, 1927: 219). En el estado actual de nuestros conocimientos, no cabe duda de que estos edificios constituían los horrea del campamento (Salido Domínguez, 2006 e.p.).

En otros campamentos, se reconocieron edificios interpretados como horrea, pero en el estado actual de nuestro conocimiento, podemos rechazar dicha hipótesis (Salido Domínguez, 2006 e.p.). Con ello, me refiero al almacén del campamento de Cáceres el Viejo (Cáceres, Extremadura) (Ulbert, 1984) o a los supuestos horrea de Atxa. Respecto al primero, desde que los trabajos realizados por Schulten fueran publicados en 1984 por G. Ulbert, las intervenciones arqueológicas han sido muy puntuales en este yacimiento (Cañas Aparicio et al., 2000). Los restos arquitectónicos corresponden a un campamento de época republicana, fechado en torno al primer tercio del siglo I a.C., aunque la datación exacta de la construcción sigue siendo incierta ${ }^{4}$. Ulbert interpretó como horreum un edificio (E), integrado en un conjunto arquitectónico (V). El

4 Schulten supuso una ocupación corta del yacimiento, en torno a los años 79-78 a.C. en relación con las campañas de Q. Caecilius Metellus Pius contra los lusitanos bajo la dirección del comandante Sertorius (Schulten, 1918). M. Beltrán Lloris propuso una datación temprana, 96-95 y 93 a.C. que relacionó con la operación victoriosa del proconsul $P$. Licinius Crassus contra los lusitanos (Beltrán Lloris, 19731974; 1976). estudio de las técnicas de construcción (canal en el interior) y el material arqueológico encontrado (proyectiles de piedra para catapulta) nos permiten negar su uso como granero. En el otro yacimiento mencionado, Atxa, se excavaron unos edificios que, en opinión de sus excavadores, estaban destinados al almacenamiento (Gil Zubillaga, 1995). Como he podido señalar recientemente (Salido Domínguez, 2006, e.p.), la ausencia de apoyos o de un dispositivo que permitiese sobreelevar el suelo y, de este modo, ventilar el producto almacenado impide interpretar como horrea estos edificios. Es muy posible que los supuestos almacenes del grupo central sirviesen como talleres o fabricae, como evidencian los materiales arqueológicos aparecidos ${ }^{5}$, la existencia de un espacio abierto y la planta en ' $U$ ' del edificio (Gil Zubillaga, 1995). Por otro lado, el análisis polínico realizado en el yacimiento indica una escasa presencia de cerealia en época romana (Gil Zubillaga, 1995).

El avance en la investigación de los campamentos coincidio con el descubrimiento de graneros militares de época altoimperial en la región septentrional de la Península Ibérica. Las campañas de excavación realizadas por Rodríguez Colmenero y otros arqueólogos desde 1975 en el campamento de Baños de Bande (Orense) confirmaron el carácter militar del yacimiento y se centraron en el estudio del cuadrante noroeste del asentamiento (retentura), donde se pusieron al descubierto los horrea. La aparición de estos edificios confirmaba que los graneros de Hispania no eran una excepción en el Imperio y el material aparecido, las técnicas empleadas en su construcción y la planta eran idénticos a los horrea excavados en otras regiones (Rodríguez Colmenero y Ferrer Sierra, 2006: 105-106).

A diferencia de otros países, la publicación de las grandes monografías dedicadas al estudio de los graneros romanos apenas tuvo repercusión en la investigación española. En 1981, se publica el

5 En el interior de este grupo central de almacenes, se hallaron restos de manipulación de plomo, así como tres pesas de plomo, herramientas y dos llaves, que invitan a pensar que en este edificio además se almacenarían materias lo suficientemente valiosas para estar guardadas bajo llave (Gil Zubillaga, 1995). 
primer artículo referido a este tema. Bajo el título Siri, granaria y horrea en la Hispania Citerior, López Pardo presenta un estado de la cuestión en el que incluye los silos excavados en la tierra de varios yacimientos y transmite, sin crítica, la información proporcionada por Schulten para los campamentos republicanos. Apenas se hace referencia a las publicaciones extranjeras, a excepción de la obra de Rickman. Este trabajo inicia una serie de investigaciones centradas en el estudio de los graneros rurales. Se analizan estructuras concretas y aisladas, como los silos de la campiña de Córdoba (Lacort Navarro, 1982) o el supuesto horreum de Carchena que Lacort fecha en época romana (Lacort Navarro, 1985), pero ni proporciona datos que confirmen su cronología ni las técnicas constructivas de las estructuras de Carchena son propias de los horrea romanos. Pocos avances presenta la publicación del libro Las villas romanas en España de 1982 de Fernández Castro que incluye estancias de villae como posibles graneros. También Dupre hace un esfuerzo por incluir los graneros de ámbito rural en el artículo Le stockage des céréales dans le bassin de l'Ebre à l'époque romaine, pero los datos cronológicos no son fiables y las interpretaciones sobre la funcionalidad de ciertas estancias son erróneas. El principal problema estriba en el desconocimiento de la bibliografía extranjera y, por tanto, de los elementos que permiten definir un edificio como granero.

Ante la ausencia de un registro arqueológico que correspondiera a graneros romanos, se interpretaron como almacenes estructuras que no cumplían esta función. Las causas que explican la ausencia de horrea en las excavaciones arqueológicas de nuestro país son múltiples. El motivo principal es el problema endémico que ha existido en la arqueología española centrada en el descubrimiento de la zona más suntuaria de la villa romana (la pars urbana) con el fin de desenterrar las salas pavimentadas con mosaicos y encontrar el material más ostentoso. Las estructuras correspondientes a la pars rustica no llamaban la atención de los arqueólogos y el desinterés provocó, a su vez, un paulatino desconocimiento del funcionamiento y técnicas de construcción de estas edificaciones al mismo tiempo que se empezaban a reconocer las diferentes salas que componían la pars urbana. Por otro lado, la utilización de materiales más modestos en los edificios de funciona- lidad rústica provocó que, en la antigüedad, muchos elementos constructivos del edificio fueran reaprovechados para la edificación de otras estructuras; en otros casos, probablemente el uso de madera ha impedido su reconocimiento en el registro arqueológico. En la actualidad, el empleo de este tipo de materiales ha supuesto el abandono de muchas construcciones que formaban parte de la villa romana e incluso su derrumbe, lo que impide su reestudio.

A principios de los años '90, un grupo de arqueólogos comienza a excavar villae romanas que integraban edificios interpretados correctamente como graneros. Se trata de las campañas de excavación realizadas en varias villae de la Lusitania dirigidas por investigadores como Cerrillo Martín de Cáceres en Monroy (Cáceres) (Cerrillo et al., 1988; 1991), Alarcão, Etienne y Mayet en Sao Cucufate (Alarcão et al., 1990), Encarnaçao y Cardoso en Freiria (Encarnaçao et al., 1992-3), Aguilar Saenz y Guichard (1993) en las villas romanas de Doña María y La Sevillana. Será precisamente Aguilar Sáenz quien publica en 1991 el estudio más metódico sobre las Dependencias con funcionalidad agrícola en las villas romanas de la Península Ibérica. Desde la publicación de este artículo, apenas se ha investigado sobre los sistemas de almacenaje de grano en las villas hispanorromanas. Los motivos que explican la ausencia de este tipo de estudios son el desinterés generalizado de los especialistas por analizar las estructuras de funcionalidad agrícola en la Península Ibérica, la paralización de numerosas excavaciones desde mediados de los años '90, la puesta en marcha de la musealización de innumerables yacimientos que marginan estas construcciones en su proyecto de visita al público y la ausencia en el registro arqueológico de edificios de este tipo que se debe al progreso de las excavaciones en ciudades en el marco del desarrollo de la arqueología de gestión o urbana. No obstante, algunos establecimientos rurales de época romana comenzaron a ser excavados y el empeño de sus excavadores permitió continuar intervenciones en yacimientos concretos. En este sentido, podemos destacar la intervención en la villa romana de Veranes (Gijón) dirigida por Fernández Ochoa y Gil Sendino (Fernández Ochoa, et al., 2004; Fernández Ochoa, Gil Sendino, 2008). El descubrimiento de un horreum perteneciente a la fase altoimperial de la villa 
romana que se mantiene en uso en época bajoimperial fomentó el interés de estos especialistas sobre este tipo de construcciones ${ }^{6}$.

\section{Perspectivas de futuro y PROPUestas DE TRABAJO}

El desarrollo de la investigación de innumerables yacimientos debe prestar atención a la importancia de este tipo de construcciones, pues constituyen el elemento clave para comprender uno de los capítulos más importantes de la economía romana. Es necesario destacar que el comercio más importante en la Antigüedad fue el transporte y distribución de los productos de primera necesidad, entre los que se encuentra el grano, y este estudio aporta las herramientas metodológicas imprescindibles que nos permiten aproximarnos al conocimiento de este tipo de transporte y comercio de época romana.

La excavación de edificios de estas características requiere un trabajo cuidadoso que permita la documentación de las fases de construcción, el registro y estudio de los materiales aparecidos en el interior, la recogida de muestras para su posterior análisis y la obtención de datos que puedan tener especial relevancia en la interpretación de una edificación con esta funcionalidad.

En primer lugar, es importante reconocer las reformas que se acometen en un edificio para determinar las fases constructivas y comprender la evolución histórica del conjunto. Son innumerables los yacimientos cuyas plantas no responden a la realidad histórica de un periodo sino de varias épocas que no han sido reconocidas en el registro arqueológico. La mayoría de los graneros excavados no fueron datados correctamente $y$, en muchas ocasiones, se adscriben al periodo de mayor desarrollo arquitectónico de una villa o aglomeración urbana. La revisión del material podría aportar nuevos datos sobre el momento de inicio de construcción de muchos edificios.

Respecto a los materiales encontrados, se estima necesario encontrar elementos de sustentación que permitan sobreelevar el pavimento interno, el principal testimonio que evidencia la existencia

6 El interés de la Dra. Fernández Ochoa por este tipo de edificaciones se ha traducido en el encargo al autor de este artículo de la realización de una tesis doctoral dedicado a este de un granero; de igual modo, la presencia de mechinales o huecos realizados en la pared o dobles muros nos indican la colocación de soportes de dicho suelo. Es necesario además recoger restos de materiales carbonizados de madera $\mathrm{o}$ clavos, así como reconocer las improntas de poste que evidencian la existencia de graneros construidos en materiales perecederos lignarios. La abundancia de documentación relativa a edificios de este tipo en otras regiones del Imperio nos indica que el uso de la madera sería siendo muy común en época romana.

La recogida de muestras de semillas y su posterior análisis ha sido un avance fundamental en este campo. La investigación arqueobotánica ha permitido determinar los productos almacenados, conocer el sistema de almacenaje y determinar las causas del incendio o abandono del edificio a partir del estado de conservación de los granos aparecidos (carbonización, germinación, etc.). Además ha posibilitado no sólo reconocer las especies introducidas por los romanos en las regiones conquistadas y la importación a larga distancia de ciertos productos, sino también obtener conclusiones muy interesantes sobre el sistema de explotación del territorio, el tipo de cultivo y la reconstrucción paleoambiental de los yacimientos. Tan importante como la documentación de restos botánicos es registrar los datos relativos a la fauna del entorno. Estudios tan completos como la tesis doctoral de Kooistra (1996) advierten de la necesidad de combinar los estudios arqueobotánicos y los resultados zooarqueológicos para comprender la relación entre la explotación agrícola de un territorio y la cría y consumo de animales, lo que permite comprender cómo varía esta relación en el tiempo y en el espacio.

Reconocidos los edificios destinados al almacenaje de grano y analizados los datos procedentes de las excavaciones, se debe proceder a la cuantificación de la capacidad máxima de almacenamiento. Como hemos podido comprobar, los diferentes especialistas que han centrado su atención en estos estudios plantearon varias propuestas que en la actualidad necesariamente deben ser cuestionadas y relativizadas. Es preciso revisar los datos aportados por autores como Gentry o

tema. Los primeros resultados fueron publicados en 2008 y esperan ser continuados en años venideros (Salido Domínguez, 2008). 
Manning para el estudio de los graneros militares y es necesario abordar el estudio del volumen de grano que podría almacenarse en los graneros rurales y urbanos. En este sentido, la información procedente de la etnoarqueología y la arqueobotánica nos obligan a examinar ciertos datos importantes para la presentación de resultados de este tipo. En primer lugar, hay que destacar que en graneros con el pavimento sobreelevado la forma de almacenar el cereal es verter a granel las semillas en espigas en la superficie del suelo, aunque se trate de cereales desnudos o vestidos, cebada o trigo $^{7}$. Los estudios arqueobotánicos indican que no toda la superficie del pavimento interno estaría cubierta de grano, pues era necesario habilitar espacios de circulación y ventilación para evitar la fermentación del grano, además es muy posible que existiesen diferentes compartimentos en el interior. Las fuentes clásicas mencionan la construcción de dichos espacios para separar los tipos de granos (Columela, De Re Rustica I 6, 9-24) y el estudio de los restos de semillas aparecidos en el interior del horreum de Amiens confirma una cierta organización interna y separación de productos en la cámara de almacenamiento (Matterne et al, 1998). Tampoco sería posible amontonar el grano hasta una altura de más de un metro (Sigaut, 1981: 168) para un amontonamiento de unos $2 \mathrm{~m}$. de extensión en su base (Matterne, 2000). El espesor medio de la capa de cereal debe ser de entre 20 y $40 \mathrm{~cm}$. (Matterne et al, 1998) para mantener las condiciones óptimas de conservación del cereal y evitar la fermentación del grano. Estos datos nos advierten de la necesidad de revisar los estudios de cuantificación de la capacidad máxima de grano almacenado en los horrea excavados, reconsiderar los datos referidos a la explotación agrícola de un territorio así como a la cantidad de cereal estimado para el consumo directo y la exportación.

Para finalizar, es indispensable realizar un corpus documental actualizado con todos los datos relativos a los horrea excavados en el occidente del Imperio romano con el fin de reconstruir la apariencia de estos edificios, destacar las semejanzas y diferencias regionales en cuanto a este tipo de construcciones y determinar la capacidad

7 Los análisis realizados demuestran que se almacenaba el cereal a granel en los horrea de South Shields (Van der Veen, 1988), Alcester (Booth, 1989), Valkenburg (Pals, Beemster y Noordam, 1989), Londres (Straker, 1984), Amiens productiva y de almacenaje en las distintas regiones. El fin último de esta investigación es obtener conclusiones interesantes sobre la explotación agrícola del territorio, la introducción y expansión de diferentes especies de cereal en las provincias que quedaron sometidas al poder de Roma, conocer los mecanismos de administración y redes comerciales, así como investigar el impacto en las comunidades locales de los procedimientos utilizados por el ejército en la obtención del grano (contratos, requisa y/o tributación) y su repercusión en la comercialización y distribución del grano y en la propia arquitectura de los graneros de las provincias del occidente del Imperio ${ }^{8}$.

\section{Bibliografía}

AGUILAR SÁENZ, A., (1991): "Dependencias con funcionalidad agrícola en las villas romanas de la Península Ibérica". Gerión (Alimenta. Estudios en Homenaje al Dr. Michel Ponsich), Anejos III, 261-279.

AGUILAR SAENZ, A. y GUICHARD, P., (1993): Villas Romaines d'Estrémadure. Doña María, La Sevillana et leur environnement. Ecole des Hautes études Hispaniques-Casa de Velázquez. Collection de la Casa de Velázquez 43. Madrid.

ALARCÃO, J., ETIENNE, R., MAYET, F., (1990): Les villas romaines de Sao Cucufate. Fouilles de Conimbriga. París.

ALCOCK, L., (1954): “Castell Collen Excavations 1954". Transactions of the Radnorshire Society 24, 62-73.

ALCOCK, L., (1955): "Castell Collen Excavations 1955: a summary account". Transactions of the Radnorshire Society 25, 46-69.

ALCOCK, L., (1956): “Castell Collen Excavations 1956: a summary account". Transactions of the Radnorshire Society 26, 10-21.

ALCOCK, L., (1957): "Castell Collen Excavations 1957". Transactions of the Radnorshire Society 27, 5-11.

ALZON, C., (1964): Problèmes relatifs à la location des entrepôts en Droit romain. París.

(Matterne et al., 1998) y en la villa de Voerendaal (Kooistra, 1996).

8 Este es un trabajo que estoy desarrollando en mi tesis doctoral, actualmente en fase de finalización. 
BABLED, H., (1892): De la cura annonae chez les Romains. París.

BELTRÁN LLORIS, M., (1973-1974): "Problemas de arqueología cacereña. El campamento romano de Cáceres el Viejo (Cáceres). Estudio numismático". Numisma 23-24, 255-310.

BIRLEY, E., (1960): Chesters Roman Fort, Northumberland. Londres.

BIRLEY, E., (1961): Research on Hadrian's Wall. Kendal.

BISHOP, M.C., (1994): Corstopitum: An Edwardian Excavation. Photographs from the 1906-14 excavations of the Roman Site at Corbridge, Northumberland. Londres.

BLECH, M., (2007): “Adolf Schulten, el Instituto Arqueológico Alemán y sus investigaciones en los campamentos romanos". En MORILLO, A. y AURRECOECHEA, J., (eds.): El ejército romano en Hispania. Guía arqueológica. León, 27-38.

BOGAERS, J. E., (1967): “Die Besatzungstruppen des Legionslagers von Nijmegen im 2. Jahrhundert nach Christus". En BIRLEY, A. R., (ed.): Studien $z u$ den Militärgrenzen Roms. Vorträge des 6. Internationalen Limeskongresses in Süddeutschland. Beihefte der "Bonner Jahrbücher” 19. Köln, 54-76.

BOOTH, P. M., (1989): "Roman store buildings at Alcester". Birmingham and Warwickshire Archaeological Society 94. Transactions for 1985-6, 63-106.

BOSANQUET, R. C., (1904): "Excavations on the line of the Roman Wall in Northumberland: The Roman Camp at Housesteads". AA 2 (25), 193299.

BREWIS, P., (1925): "Roman Rudchester: Report on excavations 1924". AA 4 (1), 93-120.

BRUNSTING, H., (1961): "Die Nijmeegse castra. Resultaten van de opgraving in 1960". Numaga 8 , 49-67.

BUCHANAN, M., (1903): "Excavation of Castlecary Fort on the Antonine Vallum". PSAS 37, 308.

BUCHANAN, M., (1905): "Report on the society's excavation of Rough Castle on the Antonine Vallum”. PSAS 39, 442-499.

BUCKLAND, P.C., (1976): The Environmental Evidence from the Church Street Roman Sewer System. Archaeology of York 14/1. Londres.
BUSANA, M. S., (2002): Architetture rurali nella Venetia romana. Le rovine circolari 3. Roma.

BUXÓ I CAPDEVILA, R., (1991): Arqueología de las plantas. La explotación económica de las semillas y los frutos en el marco mediterráneo de la Península Ibérica. Barcelona.

CAGNAT, R., (1916): “L'annone d'Afrique”. Mémoires de l'institut nacional de France, Académie des inscriptions et belles letres 40, 247-277.

CAÑAS APARICIO, R., GONZÁLEZ FERNÁNDEZ, M. L. y ABASOLO, J. A., (2000): "Proyecto de excavación y adecuación del yacimiento de Cáceres el Viejo y centro de interpretación en Cáceres". En RASCÓN MARQUÉS, S., (ed.): Ciudad, Arqueología y Desarrollo: la musealización de los yacimientos arqueológicos. Alcalá de Henares, 281-286.

CERRILLO MARTÍN DE CACERES, E., ALVARADO GONZALO, M., CERRILLO MARTÍN DE CACERES, F. J., HERRERA GARCIA, G. y GONZÁlEZ LUCEÑO, M ${ }^{\mathrm{a}}$ A., (1988): "Excavaciones en la villa romana de Monroy (Cáceres). 1981-1985)", Extremadura Arqueológica 1, 167-186.

CERRILlO, E., DE CÁCERES, HERRERA, G., MOLANO, J., DE ALVARADO, A., CASTILA, J., HERNÁNDEZ LÓPEZ, M., (1991): "Excavaciones arqueológicas en la villa romana de «Los Términos», Monroy (Cáceres). Actuaciones y propuestas de futuro. (19841990)", Extremadura Arqueológica 4 (I Jornadas de Prehistoria y Arqueología en Extremadura, 1986-1990), 379-386.

CHASTAGNOL, A., (1960): La préfecture urbaine à Rome sous le Bas-Empire. París.

CHRISTISON, D. y ANDERSON, J., (1901): "Excavation of the Roman camp at Lyne, Peeblesshire, undertaken by the Society of Antiquaries of Scotland in 1901". PSAS 35 (1900-1901), 154-186.

CLARKE, J., (1933): The Roman fort at Cadder (near Glasgow). Glasgow.

CUNLIFFE, B., (1968): Fifth report on the excavations of the Roman fort at Richborough, Kent. Report of the Research Committee of the Society of Antiquaries of London 23. Oxford.

CUNLIFFE, B., (1971): Excavations at Fishbourne 1961-1969. Volume I: The Site. Reports of the 
Research Committee of the Society of Antiquaries of London 26. Londres.

CUNLIFFE, B., DOWN, A.G. y RUDKIN, D.J., (1996): Chichester Excavations IX. Excavations at Fishbourne 1969-1988. Chichester.

DAL MONTE, G., (1956): "La presenza di insetti dei granai in frumento trovato negli scavi di Ercolano". Redia 41, 23-28.

DEMAREZ, J.-D., (1987): "Les bâtiments à fonction économique dans le fundi de la Provincia Belgica". Amphora 50, 1-36.

DOBSON, M., (2008): The army of the Roman Republic: the second centruy BC, Polybius and the camps at Numantia, Spain. Oxford.

DUPRÉ, N., (1991): "Le stockage des céréales dans le bassin de l'Ebre à l'époque romaine". Gerión (Alimenta. Estudios en Homenaje al Dr. Michel Ponsich), Anejos III, 205-217.

DURLIAT, J., (1990): De la ville antique à la ville byzantine le problème des subsistances. Collection de l'École Française de Rome 136. Roma.

EIDAM, H., (1905): Das Kastell Theilenhofen. ORL 71a. Heidelberg.

ENCARNAÇAO, J., D'. y CARDOSO, G., (1992-3): "A villa romana de Freiria e o seu enquadramento rural". Studia Historica. Historia Antigua 1011, 203-217.

EVELYN-WHITE, H.G., (1914): "Excavations at Castell Collen, Llandrindod Wells". Archaeologia Cambrensis 14, 1-58.

FABRICIUS, E., (1904): Das Kastell Urspring. ORL 66a. Heidelberg.

FABRICIUS, E., KOHL, W. y TRÖLTSCH, J., (1906): Das Kastell Weissenburg. ORL 72. Heidelberg.

FERNÁNDEZ CASTRO, M. C., (1982): Las villas romanas en España. Madrid.

FERNÁNDEZ OCHOA, C. y MORILLO CERDÁN, A., (2002): "Entre el prestigio y la defensa: la problemática estratégico-defensiva de las murallas tardorromanas en Hispania". Gladius. Anejos $5,577-589$.

FERNÁNDEZ OCHOA, C., GIL SENDINO, F., y OREJAS SACO DEL VALLE, A., (2004): "La villa romana de Veranes. El complejo rural tardorromano y propuesta de estudio del territorio". AEspA 77 (189-190), 197-220.
FERNÁNDEZ OCHOA, C., GIL SENDINO, F., (2007): "La etapa final de Roma en Hispania: la villa de Veranes (Gijón, Asturias)". En FERNÁNDEZ-TRESGUERRES, J., (coord.): Astures y romanos: Nuevas perspectivas. Oviedo, 133-148.

FERNÁNDEZ OCHOA, C. y GIL SENDINO, F., (2008): "La villa romana de Veranes (Gijón, Asturias) y otras villas de la vertiente septentrional de la cordillera cantábrica". En FERNÁNDEZ OCHOA, C., GARCÍA-ENTERO, V. y GIL SENDINO, F., (eds.): Las villae tardorromanas en el occidente del Imperio. Arquitectura y función. IV Coloquio Internacional de Arqueología en Gijón. Gijón, 435-479.

FLORIANI SQUARCIAPINO, M., (1996): "Russell Meigs e Ostia”. En GALLINA ZEVI, A., y CLARIDGE, A., (eds.): 'Roman Ostia' revisited: archaeological and historical papers in memory of Russell Meiggs. Londres/Ostia, 13-18.

GAST, M. y SIGAUT, F., (dirs.) (1979): Les techniques de conservation des grains à long terme 1 . Leurs rôles dans la dynamique des systèmes de culture et des sociétés. París.

GAST, M. y SIGAUT, F., (dirs.) (1981): Les techniques de conservation des grains à long terme 2 . Leurs rôles dans la dynamique des systèmes de culture et des sociétés. París.

GAST, M., SIGAUT, F., BEUTLER, C., (dirs.) (1985): Les techniques de conservation des grains à long terme 3. Leurs rôles dans la dynamique des systèmes de culture et des sociétés. París.

GEBHARDT, E., (1881): Studien über das Verpflegungswesen von Rom und Constantinopel in der späteren Kaiserzeit. Dorpat.

GENTRY, A. P., (1976): Roman military stone-built granaries in Britain. BAR 32. Oxford.

GIL ZUBILLAGA, E., (1995): Atxa, poblado indígena y campamento militar romano. Memorias de yacimientos alaveses 1, Vitoria-Gasteiz, 1995.

GILLAM, J. P., (1961): "Excavations at Haltonchesters 1961". Durham University Gazette 2 (9), 6.

GÓMEZ-PANTOJA, J. y MORALES HERNÁNDEZ, F., (2002): "Sertorio en Numancia: Una nota sobre los campamentos de la Gran Atalaya". En MORILLO CERDÁN, A., (coord.): Arqueología Militar Romana en Hispania, Anejos Gladius 5. Madrid, 303-310. 
GRÖNKE, E., (1997): Das römische Alenkastell Biricianae in Weissenburg i. Bay. Die Grabungen von 1890 bis 1990. Limesforschungen 25. Mainz.

HAVERFIELD, F., (1910): Military aspects of Roman Wales. Londres.

HEIMBERG, U., (2002-2003): "Römische Villen an Rhein und Maas". BJ 202-203, 57-148.

HELBAEK, H., (1964): “The Isca grain, a Roman plant introduction in Britain". The New Phitologist 63 (2), 158-164.

HILDEBRANT, H. J., (1979): "Die Römerlager von Numantia. Datierung anhand der Münzfunde". MM 20, 238-271.

HIRSCHFELD, O., (1870): “Die Getreideverwaltung in der römischen Kaiserzeit". Philologus 29, 1-96.

HOGG, A.H.A., (1968): "Pen Llystyn: a Roman fort and other remains". Archaeological Journal 125, 101-192.

JACOBI, H., (1897): Das Römerkastell Saalburg bei Homburg vor der Höhe; nach den Ergebnissen der Ausgrabungen und mit Benutzung der hinterlassenen Aufzeichnungen des königl. Konservators Obersten A. von Cohausen. Homburg vor der Höhe.

JACOBI, H., (1906): Das Kastell Kapersburg. ORL 12. Heidelberg.

JACOBI, H., (1937): Das Kastell Saalburg. ORL 11. Heidelberg.

JAIDI, H., (1990): L'Afrique et le blé de Rome aux IVème et Vème siecles. Publications de la Faculté des sciences humaines et sociales de Tunis. Série 4, Histoire, 37. Tunis.

JAMES, F. T., (1906): "Roman remains: Penydarren Park, Merthyr Tydfil". Archaeologia Cambrensis 6, 193-208.

KENWARD, H. K. y WILLIAMS, D., (1979): "Biological Evidence from the Roman warehouses in Coney Street". Archaeology of York 14 (2), 45-100.

KNÖRZER, K.-H., (1970): Novaesium 4. Römerzeitliche Pflanzenfunde aus Neuss. Bandzählung 4, Limesforschungen 10. Berlín.

KNOWLES, W. H. y FORSTER, R.H., (1910): The Romano British site of Corstopitum (Corbridge, Northumberland). An Account of the excavation during 1909, conducted by the Committee of the Corbridge Excavation Fund. Londres.
KOENEN, C., (1904): "Beschreibung von Novaesium”. BJ 111-112, 97-242.

KOHL, W., (1896): Das Kastell Ruffenhofen. ORL 68. Heidelberg.

KOOISTRA, L. I., (1996): Borderland Farming. Possibilities and Limitations of Farming in the Roman Period and Early Middle Ages between the Rhine and Meuse. Amersfoort.

KRAKAUER, G., (1874): Das Verpflegungswesen der Stadt Rom in der späteren Kaiserzeit. Leipzig.

KRIER, J. y WAGNER, R., (1983): “Römisches Landgut bei Wasserbillig/Langsur "An de Fréinen"”. Hémecht 35, 211-276.

LACORT NAVARRO, P.J., (1982): "Sobre las construcciones romanas del Carchena (término municipal de Castro del Río, Córdoba)". Habis 13, 171-188.

LACORT NAVARRO, P.J., (1985): “Cereales en Hispania Ulterior: silos de época ibero-romana en la campiña de Córdoba". Habis 16, 363-386.

LAUR-BELART, R., (1935): Vindonissa: Lager und Vicus. Römisch-Germanische Forschungen 10. Berlín/Leipzig.

LEPETZ, S., y MATTERNE, V., (2003): “Élevage et agriculture dans le Nord de la Gaule durant l'époque gallo-romaine: une confrontation des donées archéozoologiques et carpologiques". RAPic 2003 (1-2), 23-35.

LÓPEZ PARDO, F., (1981): "Siri, granaria y horrea en Hispania Citerior". AEspA 54, 245-254.

LUIK, M., (2006): Renieblas, Lager V: die Ergebnisse der archäologisch-topographischen Vermessungen der Jahre 1997 bis 2001. Mainz am Rhein.

MACDONALD, G. y PARK, A., (1906): The Roman forts on the Bar Hill, Dumbartonshire. Glasgow.

MACDONALD, G. y CURLE, A. O., (1928-1929): "The Roman fort at Mumrills, near Falkirk". PSAS 63, 396-575.

MANNING, W. H., (1975): "Roman military timber granaries in Britain". SJ 32, 105-129.

MARIN, B., y VIRLOUVET, C., (dirs.) (2003a): Nourrir les cités de Méditerranée antiquité-temps modernes. París.

MATTERNE, V., (2001): Agriculture et alimentation végétale durant l'âge du Fer et l'époque galloromaine en France septentrionale. Montagnac. 
MATTERNE, V., YVINEC, J.-H., GEMEHL, D. y RIQUIER, Ch., (1998): "Stockage de plantes alimentaires et infestation par les insectes ans un grenier incendié de la fin du IIe siècle après J.-C. à Amiens (Somme)". RAPic 1998 (3-4), 93-122.

MEIGGS, R., (1960): Roman Ostia. Oxford.

MEIGGS, R., (1982): Trees and timber in the ancient Mediterranean world. Oxford.

METTLER, A., (1902): Das Kastell Benningen. ORL 58. Heidelberg.

METZLER, J., ZIMMER, J. y BAKKER, L., (1981): Ausgrabungen in Echternach. Luxemburgo.

MILLER, S.N., (1922): The Roman Fort at Balmuildy (Summerston, near Glasgow) on the Antonine wall. Glasgow.

MILLER, S.N., (1928): The Roman Fort at Old Kilpatrick. Glasgow.

MORA, G., (2007): "La arqueología militar romana en España: historia de la investigación”. En MORILLO, A. y AURRECOECHEA, J., (eds.): El ejército romano en Hispania. Guía arqueológica. León, 11-26.

MORALES HERNÁNDEZ, F., (2007): "Circumvallatio de Numantia". En MORILLO, A. y AURRECOECHEA, J., (eds.): El ejército romano en Hispania. Guía arqueológica, 263-276.

MORILLO CERDÁN, A., (1991): "Fortificaciones campamentales de época romana en España". AEspA 64, 135-190.

MORILLO CERDÁN, A., (2003): "Los establecimientos militares temporales: conquista y defensa del territorio en la Hispania republicana". En CADIOU, F., HOURCADE, D. y MORILLO CERDAN, A., (ed.): Defensa y territorio en Hispania de los Escipiones a Augusto (espacios urbanos y rurales, municipales y provinciales). León, 41-80.

MORILLO CERDÁN, A., (2006b): “Abastecimiento y producción local en los campamentos romanos de la región septentrional de la Península Ibérica”. En MORILLO CERDÁN, A., (ed.): Arqueología militar romana en Hispania II: producción y abastecimiento en el ámbito militar. León, 33-74.

MORILLO CERDÁN, A., (2007): "El ejército romano en España”. En MORILLO, A. y AURRECOECHEA, J., (eds.): El ejército romano en Hispania. Guía arqueológica. León, 87-112.
MORILLO CERDÁN, A., (coord.) (2002): Arqueología Militar Romana en Hispania. Anejos Gladius 5. Madrid.

MORILLO CERDÁN, A., (ed.) (2006a): Arqueología militar romana en Hispania II: producción y abastecimiento en el ámbito militar. León.

MORILLO, A. y AURRECOECHEA, J., (eds.) (2007): El ejército romano en Hispania. Guía arqueológica. León.

MORRIS, P., (1979): Agricultural Buildings in Roman Britain. BAR 70. Oxford.

MURRAY, O., (1996): "Russell Meiggs, practical historian”. En GALLINA ZEVI, A., y CLARIDGE, A., (1996): 'Roman Ostia' revisited: archaeological and historical papers in memory of Russell Meiggs. Londres/Ostia.

PALS, J.P., BEEMSTER, V. y NOORDAM, A., (1989): "Plant remains from the Roman castellum Praetorium Agrippinae near Valkenburg (prov. of Zuid-Holland), Archäobotanik". Dissertationes Botanicae 133, 117-134.

PAPI, E. y MARTORELLA, F., (2007): "Il grano della Tingitana". En PAPI, E. (ed.): Supplying Rome and the Empire. The Proceedings of an International Seminar held at Siena-Certosa di Pontignano on may 2-4, 2004 on Rome, the provinces, production and distribution. JRA Supplementary Series 69. Portsmouth, 85-96.

PETCH, D. F. y THOMPSON, F., (1959): "Excavations in Commonhall Street, Chester, 1954-6: the granaries of the legionary fortress of Deva". J. Chester A. S. 46, 33-60.

PETCULESCU, L., (1987): "Roman Military Granaries in Dacia”. SJ 43, 66-76.

PETRIKOVITS, H. V., (1956): "Neue Forschungen zur römerzeitlichen Besiedlung der Nordeifel". Germania 34, 99-125.

PITTS, L.F., JOSEPH, J.K.S. y COWIE, T., (1985): Inchtuthil: the Roman legionary fortress excavations 1952-65. Britannia monograph series 6. Gloucester.

QUESADA SANZ, F., (2008): Armas de Grecia y Roma forjaron la historia de la Antigüedad clásica. Madrid.

REUTER, K., (1871): Zur Geschichte des Römischen Wiesbadens. II. Das Römer-Castell bei Wiesbaden. Annalen des Vereins für Nassauische Alterthumskunde und Geschichtsforschung 5 (2). 
Wiesbaden. RITTERLING, E., (1909): Das Kastell Wiesbaden. ORL 31. Heidelberg.

REVELLO, P., (1937): Das Kastell Hüfingen. ORL 62a. Heidelberg.

REYNOLDS, P.K.B., (1938): Excavations on the site of the Roman Fort of Kanovium at Caerhun, Caenarvonshire. Collected reports on the excavations of the years 1926-1929 and on the pottery and other objects found. Cardiff.

RICHMOND, I.A., (1934): "The Roman fort at South Shields". AA 11 (4), 83-102.

RICHMOND, I.A., (1953): The Roman fort at South Shields: a guide. South Shields.

RICHMOND, I.A., (1968): Hod Hill. 2, Excavations carried out between 1951 and 1958 for the Trustees of the British Museum. Londres.

RICHMOND, I.A. y McINTYRE, J., (1939): “The Agricolan fort at Fendoch". PSAS 73, 110-154.

RICHMOND, I.A., (1959): "The Agricolan legionary fortress at Inchtuthil". En VV.AA., LimesStudien. Vorträge des 3. Internationalen LimesKongresses in Rheinfelden, Basel 1957. Basel, 152-155.

RICKMAN, G., (1971): Roman granaries and store buildings. Cambridge.

RICKMAN, G., (1980): The corn supply of Ancient Rome. Oxford.

RITTERLING, E., (1913): Das Frührömische Lager bei Hofheim im Taunus. Annalen des Vereins für Nassauische Altertumskunde und Geschichtsforschung. Wiesbaden.

RITTERLING, E., (1937): Das Kastell Nieder-Bieber. ORL 1a. Heidelberg.

RODRÍGUEZ COLMENERO, A. y FERRER SIERRA, S., (dirs.) (2006): Excavaciones arqueológicas en Aqvis Qverquennis. Actuaciones en el campamento romano (1975-2005). Anejos de Larouco 4. Lugo.

ROSSITER J.J., (1978): Farm building in Roman Italy. BAR 52. Oxford.

SALIDO DOMINGUEZ, P.J., (2003-2004) "La documentación literaria aplicada al registro arqueológico: las técnicas de construcción de los graneros romanos rurales". Espacio, Tiempo y Forma. Serie I: Prehistoria y Arqueología 16-17, 463-478.
SALIDO DOMINGUEZ, P.J., (2006) (e.p.): "Los graneros militares romanos en Hispania". $X X$ Congreso Internacional de Estudios sobre la Frontera Romana. 4-11 de Septiembre de 2006, León.

SALIDO DOMINGUEZ, P.J., (2008): "Los sistemas de almacenamiento y conservación de grano en las villae hispanorromanas". En FERNÁNDEZ OCHOA, C., GARCÍA-ENTERO, V. y GIL SENDINO, F., (eds.): Las villae tardorromanas en el Occidente del Imperio. Arquitectura y función. IV Coloquio Internacional de Arqueología de Gijón. Gijón, 693-706.

SANMARTI, E., (1992): "Nouvelles donées sur la chronologie du camp de Renieblas V à Numance (Soria, Castilla-León, Espagne)". Documents d'Archéologie Méridionale 15, 417-430.

SCHÖNBERGER, H., (1963-1964): “Augusteisches Lager Rödgen, Grabung 1963”. SJ 21, 95-108.

SCHULTEN, A., (1914): Die Ergebnisse der Ausgrabungen 1905-1912 Numantia I. Die Keltiberer und ihre Kriege mit Rom. München.

SCHULTEN, A., (1927): Die Ergebnisse der Ausgrabungen 1905-1912 Numantia III. Die Lager des Scipio. München.

SCHULTEN, A., (1929): Die Ergebnisse der Ausgrabungen 1905-1912 Numantia IV. Die Lager bei Renieblas. München.

SCHUlTEN, A., (1931): Die Ergebnisse der Ausgrabungen 1905-1912 Numantia II. Die Stadt Numantia. München.

SIGAUT, F., (1981): "Identification des techniques de conservation et de stockage des grains". En GAST, M, y SIGAUT, F., (dirs.): Les techniques de conservation des grains à long terme 3. Leurs rôles dans la dynamique des systèmes de culture et des societies. París, 156-180.

SIGAUT, F., (1988-1991): “A method for identifying grain storage techniques and its application for European Agricultural History". Tools \& Tillage 6, 3-32.

SIMPSON, F.G. y RICHMOND, I.A., (1936): “The Roman fort on the Stanegate and other remains at Old Church, Brampton". CW 2 (36), 172-182.

STEVENSON, G. H. y MILLER, S. N., (1912): "Report on the excavations at the Roman Fort of Cappuk, Roxburghshire”. PSAS 46, 446-483. 
STIEREN, A., (dir.) (1943): Die Funde von Haltern seit 1925 bis 1928. Bodenaltertümer Westfalens 6. Münster.

STRAKER, V., (1984): "First and second century carbonised cereal grain from Roman London". En VAN ZEIST, W. y CASPARIE, W. A. (eds): Plants and Ancient Man: Studies in palaeoethnobotany. Rotterdam/Londres, 323-329.

TENGSTRÖM, E., (1975): Bread for the people. Acta Instituti Romani Regnio Sueciae $8^{\circ}, 12$. Estocolmo.

ULBERT, G., (1984): Cáceres el Viejo. Ein spätrepublikanisches Legionslager in SpanischExtremadura. Madrider Breitäge band 11. Mainz am Rhein.

VAN DER VEEN, M., (1988): “Carbonised grain from a Roman granary at South Shields, North East England”. En KÜSTER, H., (ed.): Der prähistorische Mensch und seine Umwelt. Forschungen und Berichte zur Vor- und Frügeschichte in Baden-Württemberg 30. Stuttgart, 353-365.

VAN OSSEL, P. y DEFGNÉE, A., (2001): Champion, Hamois: une villa romaine chez les Condruses: archéologie, environnement et économie d'une exploitation agricole antique de la Moyenne Belgique. Etudes et documents. Archéologie 7. Namur.

VV.AA., (1994): Le ravitaillement en blé de Rome et des centres urbains des débuts de la République jusqu'au Haut Empire. Actes du colloque international organisé par le Centre Jean Bérard et I'URA 994 du CNRS. Collection Centre Jean Bérard 11; Collection de l'École française de Rome 196; Nápoles/Roma.

WARD, J., (1903): The Roman fort of Gellygaer in the county of Glamorgan excavated by the Cardiff Naturalists Society in Glamorgan. Londres.

WHEELER, R.E.M., (1923): Segontium and the Roman occupation of Wales. Y Cymmrodor. The magazine of the honourable Society of Cymmrodorion 33. Londres, 32-33.

WINKELMANN, F., (1901): Das Kastell Pfunz. ORL 73. Heidelberg.

WOLFF, G., (1902): Das Kastell Okarben. ORL 25a. Heidelberg. 


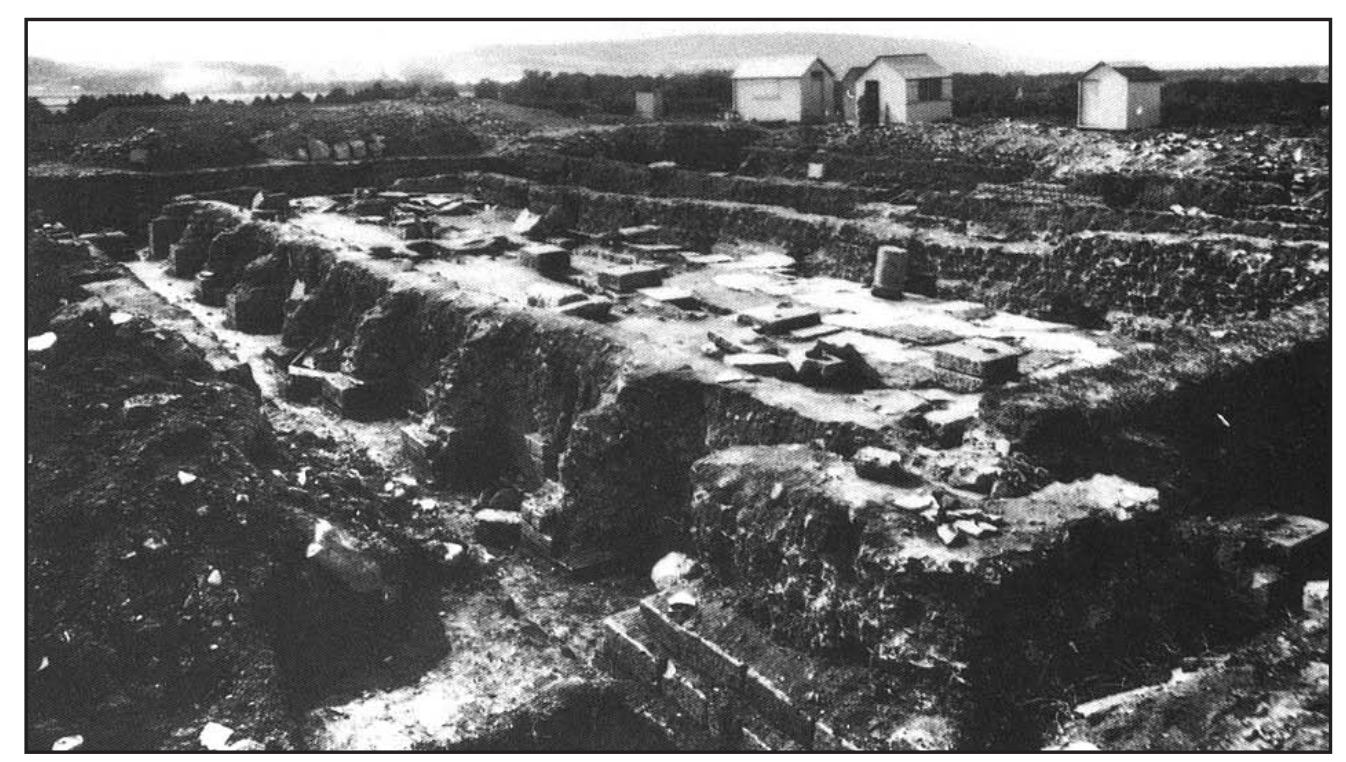

Imagen 1. Fotografía de 1909 que muestra los graneros del campamento romano de Corbridge (Northumberland) excavados en 1908, según Bishop (1994): fig. 21, Corbridge Excavation Fund.

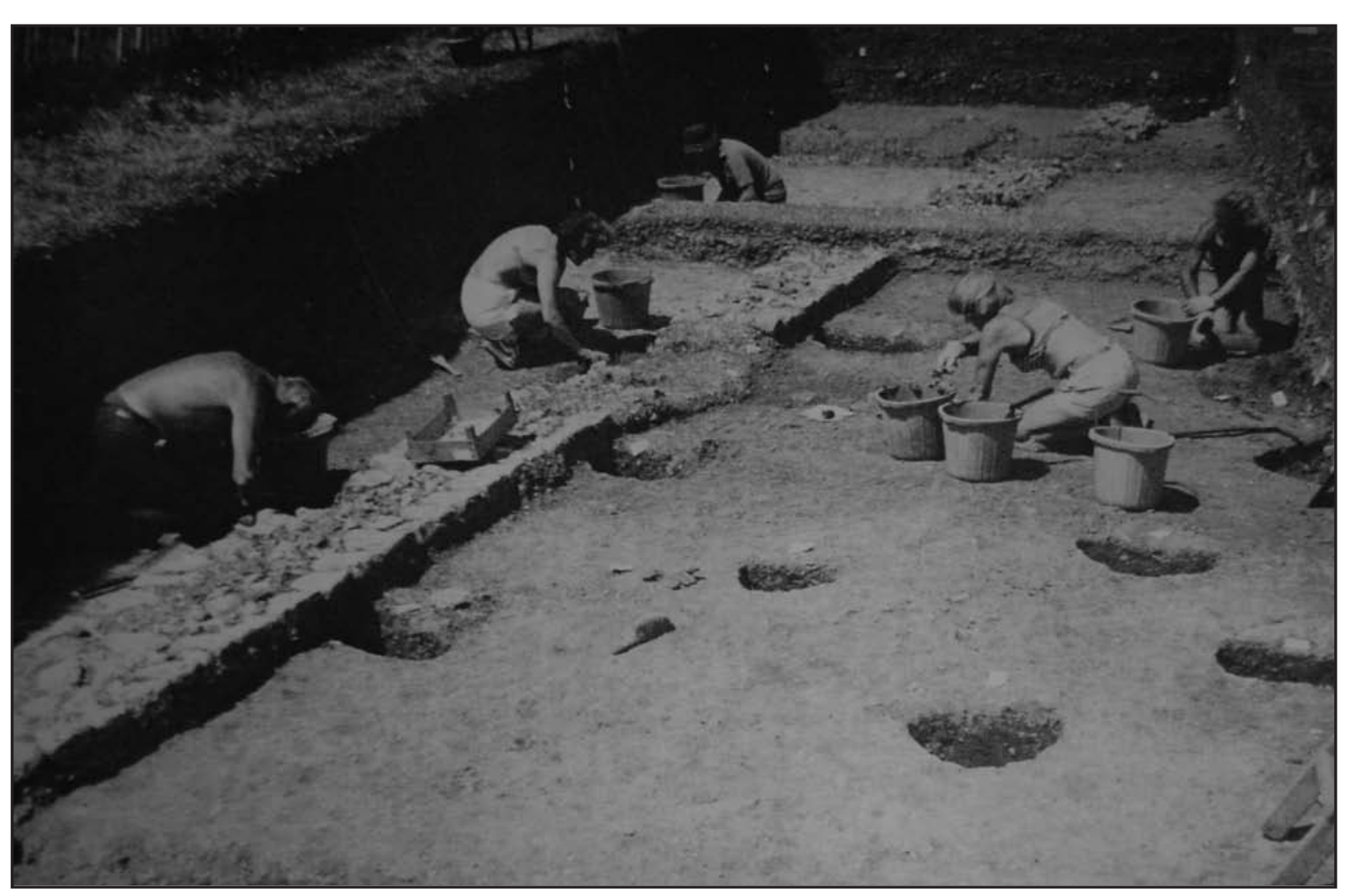

Imagen 2. Campaña de excavación arqueológica de 1983 en el interior del asentamiento militar de Fishbourne (West Sussex). Se muestra el proceso de excavación de las improntas de los postes de madera del granero de la primera fase, según Cunliffe, Down y Rudkin (1996): fig. 4. 


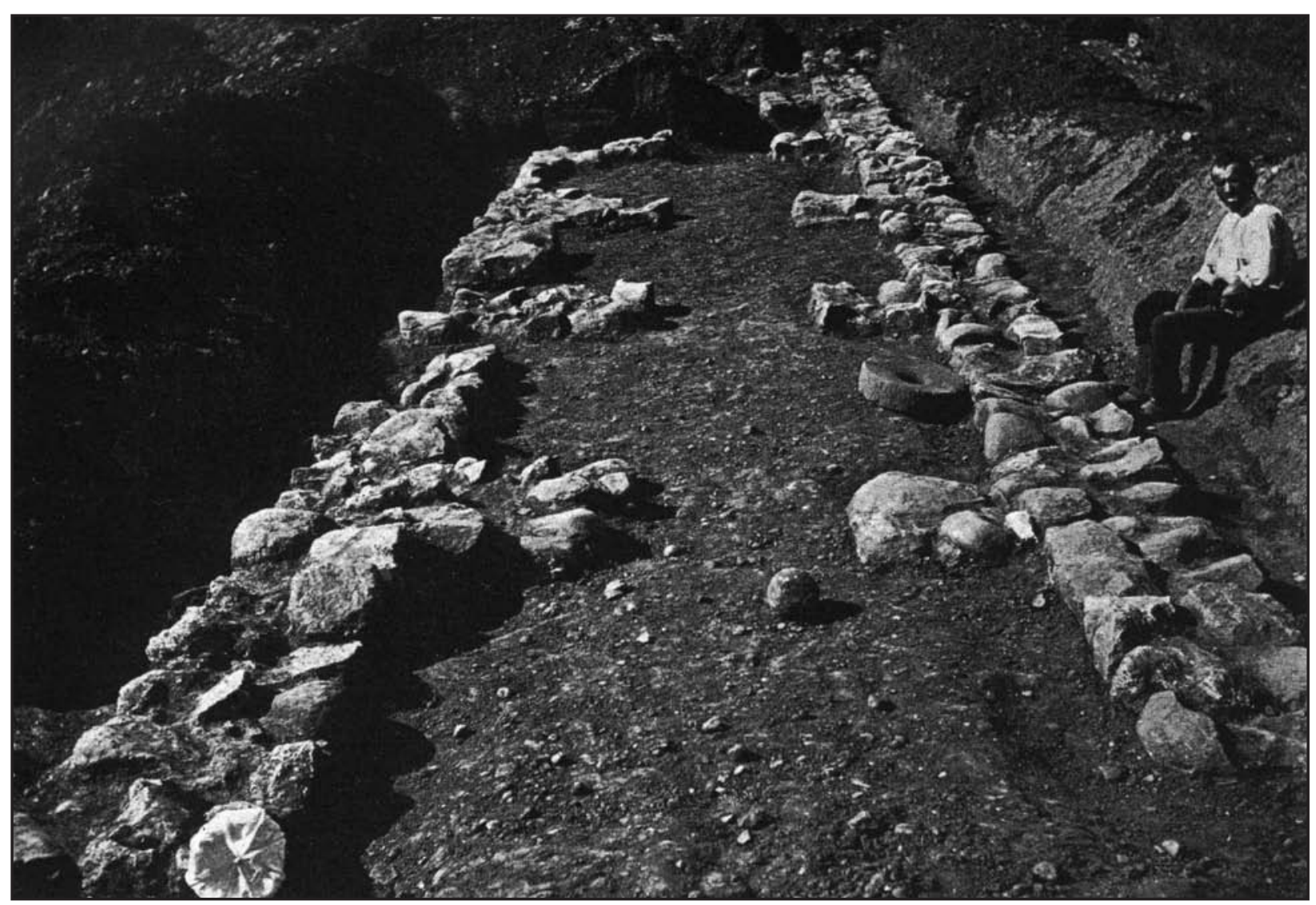

Imagen 3. Excavación de los horrea del campamento de época republicana de Castillejo (Soria), según Schulten (1927): fig. 25. 